\title{
MiRNA-621 exerts tumor suppressor function in gastric adenocarcinoma by targeting AURKA/GSK-3 $\beta$ pathway
}

\author{
Xiao Han ${ }^{1}$, Hongxue Liu² ${ }^{2}$, Xiaojun Tang ${ }^{1}$ and Yao Zhao ${ }^{1}$ \\ 'Department of Gastrointestinal Surgery, the Affiliated Huaian No.1 People's Hospital of Nanjing Medical University, Huai 'an, Jiangsu Province, \\ 223001, China; 'Department of Obstetrics, the Affiliated Huaian No.1 People's Hospital of Nanjing Medical University, Huai 'an, Jiangsu Province, \\ 223001, China
}

\begin{abstract}
Gastric adenocarcinoma is a major challenge to human health worldwide. Abnormal expression of miR-621 was found in many types of cancer. This research aimed to investigate the effects and detailed molecular mechanisms of miR-621 on gastric adenocarcinoma progression. The present study first showed that miR-621 was downregulated in gastric cancer patients, and its expression level was correlated with tumor size. MiR-621 overexpression inhibited viability, colony formation and proliferation of gastric cancer cells. AURKA was identified as a direct target of miR-621. AURKA knockdown induced decrease of $\mathrm{p}-\mathrm{GSK}-3 \beta / \mathrm{GSK}-3 \beta$ ratio and increase of $p$ - $\beta$-catenin/ $\beta$-catenin ratio which confirmed that AURKA positively regulated GSK-3 $\beta$ phosphorylation. AURKA knockdown also inhibited proliferation of gastric adenocarcinoma cells. AURKA expression was negatively correlated with miR-621 level. In addition, AURKA overexpression reversed the effect of miR-621 on the growth of cancer cells. Taken together, our results suggest that miR-621 is an important tumor suppressor in gastric cancer and could be a promising target for the cancer treatment.
\end{abstract}

Keywords: MiRNA-621; gastric adenocarcinoma; AURKA/GSK-3 $\beta$ pathway.

Received: 17 August, 2020; revised: 12 October, 2020; accepted: 30 October, 2020; available on-line: 25 February, 2021

$\square$ e-mail: liuhongxue12345@163.com

Abbreviations: AURKA, Aurora kinase A; BrdU, 5-Bromo-2'-deoxyuridine; DMSO, Dimethyl Sulfoxide; GCA, gastric cardia adenocarcinoma; GSK, Glycogen synthase kinase; JAK, Janus kinase; MTT, Thiazolyl blue tetrazolium bromide; PCR, Polymerase chain reaction; UTR, Untranslated region

\section{INTRODUCTION}

Gastric cancer is a major challenge to human health worldwide due to its high incidence and mortality. More than $95 \%$ of the tumors are adenocarcinomas, originating from the deterioration of gastric gland cells (Molina-Castro et al., 2017). Due to the late diagnosis, the average 5-year survival rate is less than $20 \%$. If the tumor can be diagnosed in early stage, the 5-year survival rate can rise to $90 \%$ (Tan, 2019; Izadpanahi et al., 2020). Therefore, it is of great significance to clarify the pathogenesis of gastric adenocarcinoma and to find new therapeutic targets and therapies.

MicroRNAs (miRNAs) are non-coding RNAs with the length of 18-22bp. MiRNAs regulate gene expression by binding to 3'-untranslated regions (3'UTR) of mRNAs (Kontomanolis et al., 2016). Many studies suggest that miRNAs play important roles from initiation to metastasis in many tumors, including gastric adenocarcinoma (Rupaimoole \& Slack, 2017). The profile of miRNAs in patients with gastric cardia adenocarcinoma (GCA) was abnormal. Juan Wang and others (Wang et al., 2018) identified five miRNAs (miR-200a3p, miR-296-5p, miR-132-3p, miR-485-3p and miR22-5p) that were up-regulated in peripheral serum of patients with GCA. Another research demonstrated that the co-expression networks of miRNAs were associated with the tumor condition. The miRNAs 100, let-7c, 125b and 99a were related to histological subtype, and miR-181 family and miR-21 were related to tumor phenotype (Yepes et al., 2016). In gastric cancer, miRNAs inhibit tumor cell proliferation, migration, and invasion through different targets (Shin \& Chu, 2014). For example, miR-375 could regulate gastric cancer cell proliferation by targeting JAK2 oncogene (Ding et al., 2010). MiR-96-5p could promote cell apoptosis via targeting ZDHHC5 gene in MGC-803 cells (Zhou et al., 2019). MiR-621 also exerts a tumor suppressor effect in many tumors, such as breast cancer, hepatocellular carcinoma, bladder cancer, and gastric cancer (Xue et al., 2016; Shao et al., 2019; Tian et al., 2019; Tao et al., 2020). However, there is still little evidence on the involvement of miR-621 in gastric cancer (Shin \& Chu, 2014). This study focuses on the level and effects of miR-621 in gastric cancer, and the detailed regulatory mechanisms.

\section{MATERIALS AND METHODS}

\section{Clinical Samples}

37 pairs of stomach samples, including 37 cancer tissue samples and paired adjacent non-cancer tissue samples, were obtained (with informed consent) from the patients with gastric adenocarcinoma who underwent surgical resection at the Affiliated Huaian No.1 People's Hospital of Nanjing Medical University. The adjacent non-cancer tissues were obtained at least 5 $\mathrm{cm}$ away from the cancer site. All cancer tissues were histologically confirmed to be gastric adenocarcinomas. Total RNA of tissue samples was isolated using Promega Super Total RNA Extraction Kit (Madison, WI, USA) according to the manufacturer's protocol. The study was approved by the Ethics Committee of the Affiliated Huaian No.1 People's Hospital of Nanjing Medical University, and the protocol was performed in accordance with the World Medical Association Declaration of Helsinki. 


\section{Cells and Culture Methods}

Human gastric cancer cell lines AGS, MKN45, SNU-1 and normal gastric epithelial cell line (GES-1) were purchased from the Type Culture Collection of the Chinese Academy of Sciences (Shanghai, China). These cells were cultured in RPMI-1640 basal medium with $10 \%$ FBS and maintained at $37^{\circ} \mathrm{C}$ in a $5 \% \mathrm{CO}_{2}$ incubator.

\section{Cell Transfection}

Vectors for miR-621 mimic, miRNA inhibitors and negative control vectors were purchased from GenePharma (Shanghai, China). The construction of miR621 expressing vector was reported previously (Shao et al., 2019). For $A U R K A$ overexpression, cDNA was amplified and inserted into the pcDNA3.1 expression vector (Invitrogen, San Diego, CA, USA). The details were reported in a previous report (Zhang et al., 2010). Transfection was performed with Lipo-2000 Reagent (Invitrogen, Carlsbad, CA, USA) following the standard protocol. Cells were collected $48 \mathrm{~h}$ after transfection.

\section{Luciferase Reporter Assays}

Cells were transfected with different combinations of miR-621 mimic, miR-621 inhibitor, respective negative control, AURKA 3'-UTR-WT and 3'-UTRMUT. The relative luciferase activities were measured by commercial dual-luciferase reporter kit (Promega, Madison, WI, USA). The details of plasmid construction were reported previously ( $\mathrm{Lu}$ et al., 2019). Fold induction was based on the relative firefly luciferase activity, normalized to Renilla luciferase.

A

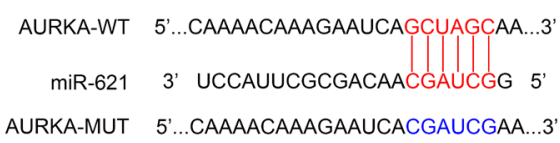

B

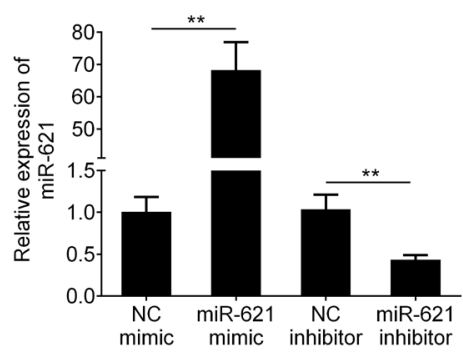

C

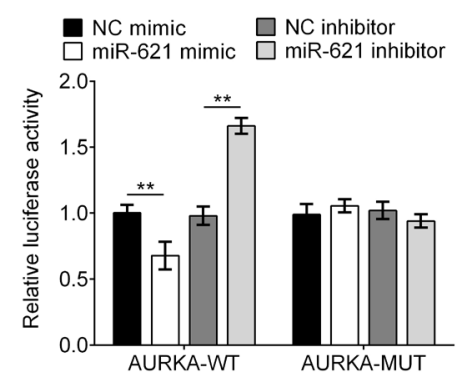

\section{Cell Viability}

Cell viability was analyzed using MTT (thiazolyl blue tetrazolium bromide) assay. Briefly, the transfected cells were incubated with MT'T solution $(0.5 \mathrm{mg} /$ $\mathrm{mL})$ at $37^{\circ} \mathrm{C}$ for $4 \mathrm{hrs}$. Then DMSO was used to dissolve the formazan, and the optical density was measured with a microplate spectrophotometer at $570 \mathrm{~nm}$.

\section{Colony Formation Assay}

The cancer cell growth was evaluated using colony formation assay. The treated cells were seeded in 12well plates at 100 cells per well. Fresh culture medium was replaced every 2 days. The number of colonies was counted from the 7 th day after seeding and cells were stained with crystal violet.

\section{Proliferation Assays}

Cancer cell proliferation assay was performed using BrdU (5-Bromo-2'-deoxyuridine) labeling according to the manufacturer's instructions. Briefly, cells were fixed on culture slides with BrdU fixation buffer, and permeabilized with $1 \%$ Triton $\times 100$. Slides were washed in PBS $3 \times 5$ minutes, followed by antigen retrieval. After antigen retrieval, samples were placed in blocking buffer and then incubated with Anti-BrdU antibody. Finally, images were acquired under a laser scanning microscope.

\section{Quantitative Reverse Transcription PCR (qRT-PCR)}

The transcription of genes was analyzed using qRTPCR. Briefly, total RNA was extracted using RNA isolation kit and cDNA was synthesized with commercial

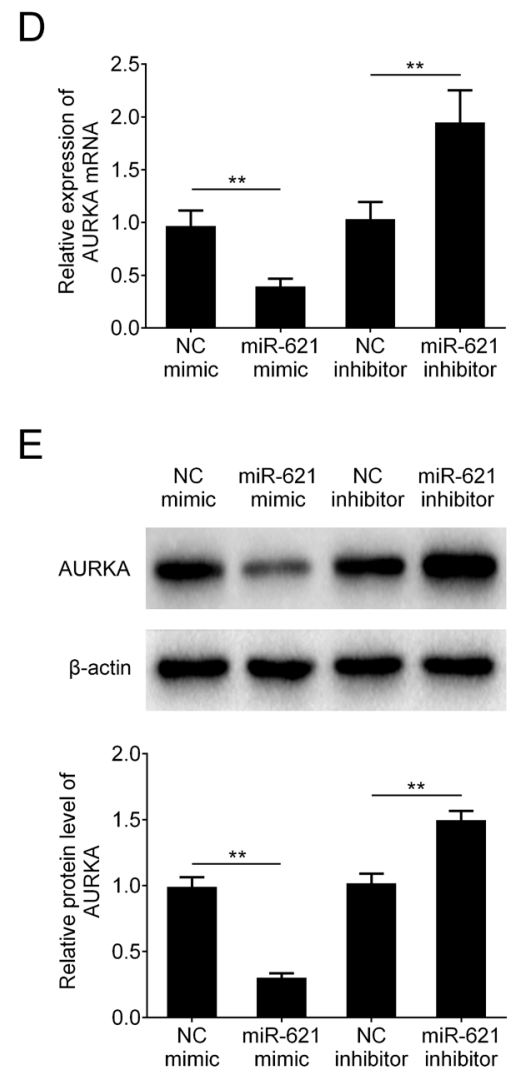

Figure 1. MiR-621 was down-regulated in human gastric adenocarcinoma. The expression of miR-621 in tumor tissues and paired adjacent noncancerous (normal) gastric tissues of patients ( $\mathrm{n}=37$ ) analyzed by qRT-PCR. ${ }^{* *} p<0.01$. 
Table 1. Relationship between the expression level of Mir-621 and the clinicopathological characteristics of gastric adenocarcinoma

\begin{tabular}{|c|c|c|c|c|}
\hline Characteristics & Number & $\begin{array}{l}\text { miR-621 } \\
\text { Low expression (< median) }\end{array}$ & $\begin{array}{l}\text { miR-621 } \\
\text { High expression ( } \geq \text { median) }\end{array}$ & $P$ value \\
\hline Number & 37 & 18 & 19 & \\
\hline Gender & & & & 0.582 \\
\hline Male & 23 & 12 & 11 & \\
\hline Female & 14 & 6 & 8 & \\
\hline Ages(years) & & & & 0.618 \\
\hline$<60$ & 18 & 8 & 10 & \\
\hline$\geq 60$ & 19 & 10 & 9 & \\
\hline Tumor size(cm) & & & & $0.002^{*}$ \\
\hline$<5$ & 20 & 5 & 15 & \\
\hline$\geq 5$ & 17 & 13 & 4 & \\
\hline Tumor invasion & & & & 0.066 \\
\hline T1 & 24 & 8 & 16 & \\
\hline $\mathrm{T} 2$ & 7 & 5 & 2 & \\
\hline T3 & 4 & 3 & 1 & \\
\hline T4 & 2 & 2 & 0 & \\
\hline Lymph node status & & & & 0.097 \\
\hline No & 20 & 9 & 13 & \\
\hline N1 & 8 & 4 & 6 & \\
\hline N2 & 5 & 3 & 0 & \\
\hline N3 & 4 & 2 & 0 & \\
\hline Histological grade & & & & 0.383 \\
\hline Well & 23 & 10 & 14 & \\
\hline Well/moderate & 12 & 7 & 5 & \\
\hline Poor & 2 & 1 & 0 & \\
\hline
\end{tabular}

${ }^{*} P<0.05$

reverse transcription system (Promega, Madison, WI). Transcription level was determined with SYBR Green reagent (Bio-Rad) with separate primers (Wu et al., 2018). Data were analyzed using the $2^{-\Delta \Delta \mathrm{Ct}}$ method with GAPDH serving as internal control.

\section{Western Blot}

Proteins were extracted using RIPA lysis buffer with proteinase and phosphatase inhibitor cocktails. Samples were separated by SDS-PAGE gels and transferred to PVDF membranes. After blocking, the target proteins were recognized by following primary antibodies at a dilution of 1:1000 to 1:2000: AURKA (CST, \#4718), GSK-3ß (CST, \#9315), Phospho-GSK$3 \beta$ (CST, \#9322), $\beta$-catenin (CST, \#8480), Phospho$\beta$-catenin (CST, \#2009). Proteins were visualized through chemiluminescence (Pierce ECL system, Thermo Fisher Scientific). Bands were quantified using Image J.

\section{Statistical Analysis}

All data were shown as mean \pm S.D. The $p$ values were calculated using one-way ANOVA (GraphPad Software, La Jolla, CA). $p<0.05$ was considered statistically significant.

\section{RESULTS}

\section{MiR-621 was down-regulated in human gastric adenocarcinoma}

To test whether the level of miR-621 in normal tissues is different than in tumor regions, real-time RT-PCR assay was performed in 37 pairs of gastric tissue samples. The result (Fig. 1) showed that miR-621 was significantly decreased in cancer tissues compared to normal tissues $(p<0.01)$. Furthermore, the level of miR-621 was also evaluated in the context of patients' clinical pathological characteristics (Table 1). Clinical pathological features included the following: gender, age, tumor size, tumor invasion and lymph node status. All patients were divided into two groups: miR-621 low expression $(n=18)$ and miR-621 high expression $(n=19)$ (median as the standard). The low-expression group tended to have larger tumors size $(p=0.002)$. However, no significant difference in the other clinical pathologic features was found.

\section{MiR-621 overexpression inhibited proliferation of gastric adenocarcinoma cells}

To investigate the effects of miR-621 in gastric adenocarcinoma, the levels of miR-621 in gastric cancer cells 


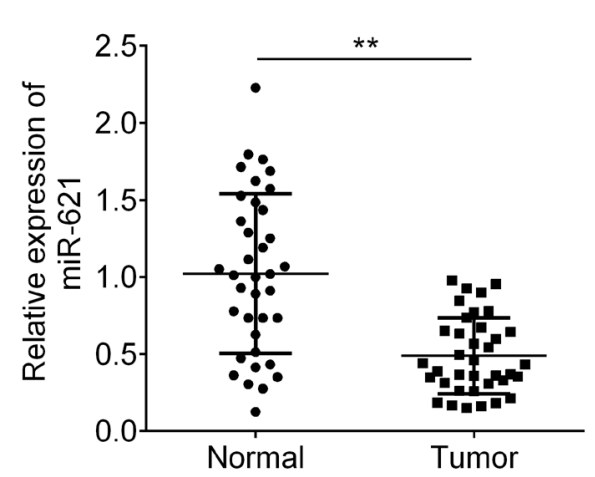

Figure 2. MiR-621 overexpression inhibited the proliferation of gastric adenocarcinoma cells.

(A) The level of miR-621 in gastric cancer cell lines (AGS, MKN45, SNU-1) and normal gastric cells (GES-1) analyzed by qRT-PCR. (B) The transfection efficiency of miR-621 in AGS and MKN45 cells measured by qRT-PCR. (C) Cell viability of AGS and MKN45 cells with miR-621 overexpression analyzed with MTT. (D) The growth of cancer cells (AGS and MKN45) with miR-621 overexpression assessed in a colony formation assay. (E) Proliferation of cancer cells (AGS and MKN45) quantified with BrdU labelling. $n=3,{ }^{* *} p<0.01$.

and normal gastric epithelial cells were analyzed. The result showed that in three gastric cancer cell lines (AGS, MKN45 and SNU-1), the expression of miR-621 was significantly lower compared with that of normal gastric mucosal cell line (GES-1) $(p<0.01)$ (Fig. 2A). To assess the function of miR-621 in gastric cancer, miR-621 overexpressing cells were obtained by transfection. The miR-

A

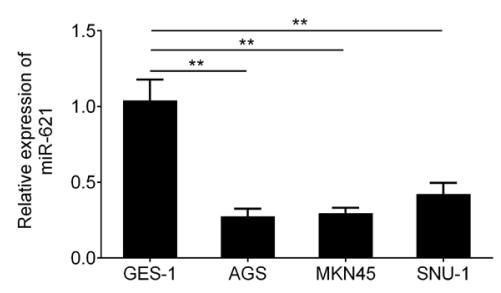

B

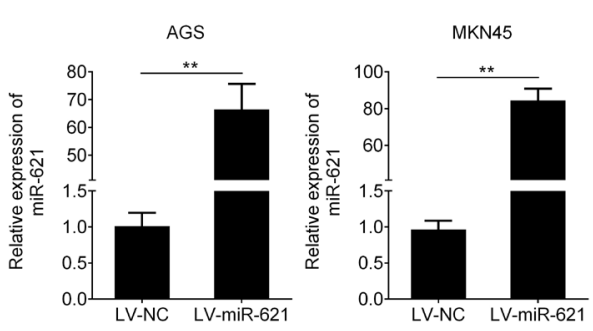

C
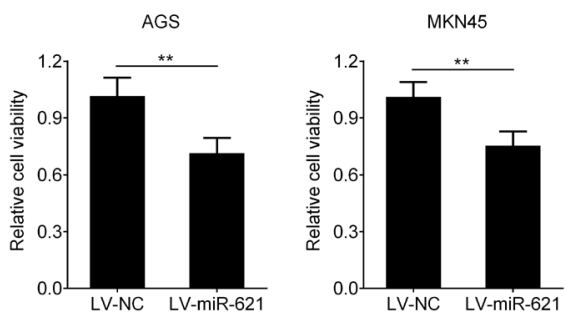

621 plasmid (LV-miR-621) or vector (LV-NC) was transfected to gastric cells (AGS and MKN45). The transfection efficiency was confirmed with RT-PCR, which showed that miR-621 overexpressing cells had a higher level of miR-621 compared to LV-NC group $(p<0.01)$ (Fig. 2B). Then cell viability, colony formation, and proliferation were analyzed in AGS and MKN45 miR-621 overexpressing cells. Cell viability (Fig. 2C), colony formation (Fig. 2D), and proliferation (Fig. 2E) were all significantly decreased after miR-621 overexpression in both cell lines $(p<0.01 v s$. LV-NC), and the effects were more pronounced in AGS cells. These results indicated that miR-621 overexpression inhibited proliferation of gastric adenocarcinoma cells.

\section{MiR-621 targeted AURKA}

To predict the target of miR-621, bioinformatics analysis (Targetscan: http://www. targetscan.org) was performed. As shown in Fig. 3A, miR-621 could bind to the 3'-UTR of AURK4 mRNA, but not when the sequence was mutated. To investigate effects of miR-621 on AURK4, miR-621 overexpressing (miR-621 mimic) or knockdown (miR-621 inhibitor) AGS cells were obtained by transfection. The transfection efficiency was confirmed with RT-PCR (Fig. 3B). The level of miR621 increased in miR-621 mimic group $(p<0.01 \mathrm{vs}$. NC mimic), and decreased in miR-621 inhibitor group $\left(p<0.01 v s\right.$. NC inhibitor). To confirm that this $3^{\prime}-$ UTR site of AURK4 was responsible for the effects of miR-621, reporter gene with 3'UTR binding site or its mutated sequence was co-transfected with miR-621 mim-

D
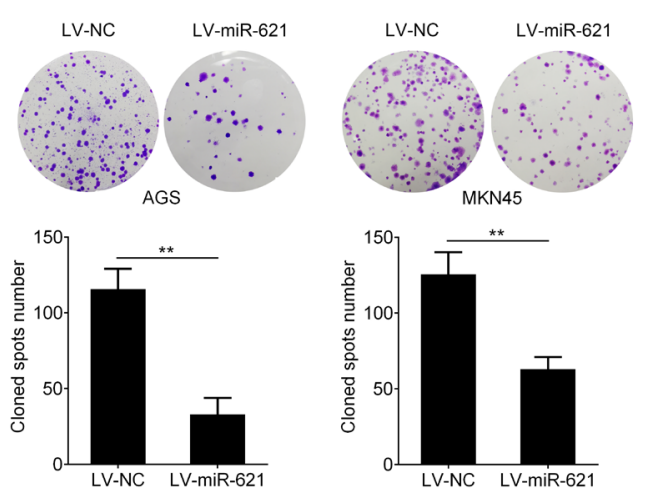

E
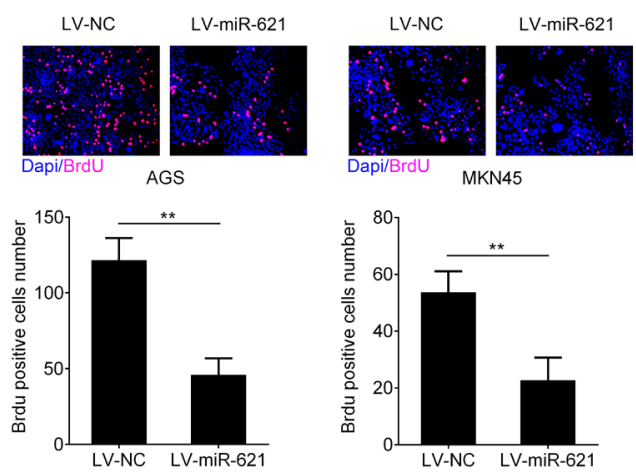

Figure 3. MiR-621 targeted AURKA

(A) The putative miR-621 binding site in AURKA 3'-UTR predicted by TargetScan. Mutation in the miR-621 binding site within the AURKA 3'-UTR is shown. (B) The transfection efficiency of miR-621 mimic or miR-621 inhibitor in cancer cells measured by qRT-PCR. (C) The relative luciferase activity of AURKA wild-type (WT) or mutant (MUT) in the cells transfected with miR-621 mimic or miR-621 inhibitor. (D) The level of AURKA in cells transfected with miR-621 mimic or miR-621 inhibitor detected with qRT-PCR. (E) The level of AURKA in cells transfected with miR-621 mimic or miR-621 inhibitor analyzed with Western blot. $n=3, * * p<0.01$. 
ic, inhibitor, or the control vector into AGS cells. The luminescence intensity of cells transfected with miR-621 (miR-621 mimic) was decreased for about 40\% $(p<0.01$ vs. NC mimic), while increased about 2 folds in miR-621 inhibitors group. This effect was not found in cells with mutated AURK4 (Fig. 3C), which indicated that this site was responsible for the negative regulation of miR-621 on AURK4. The level of AURK4 mRNA (Fig. 3D) decreased in miR-621 overexpressing cells, and increased in miR-621 knockdown cells. The protein level (Fig. 3E) of AURK4 was in accordance with its mRNA level. These results indicated that AURK4 was a target of miR-621.

A
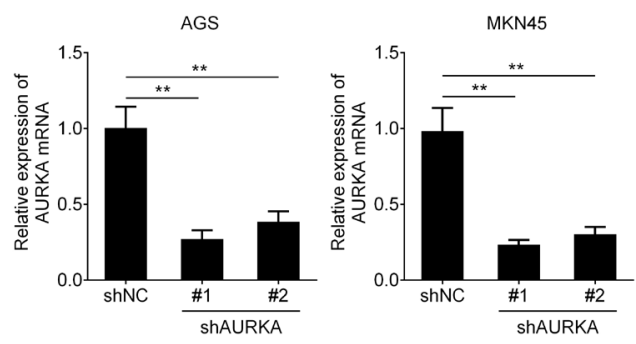

B
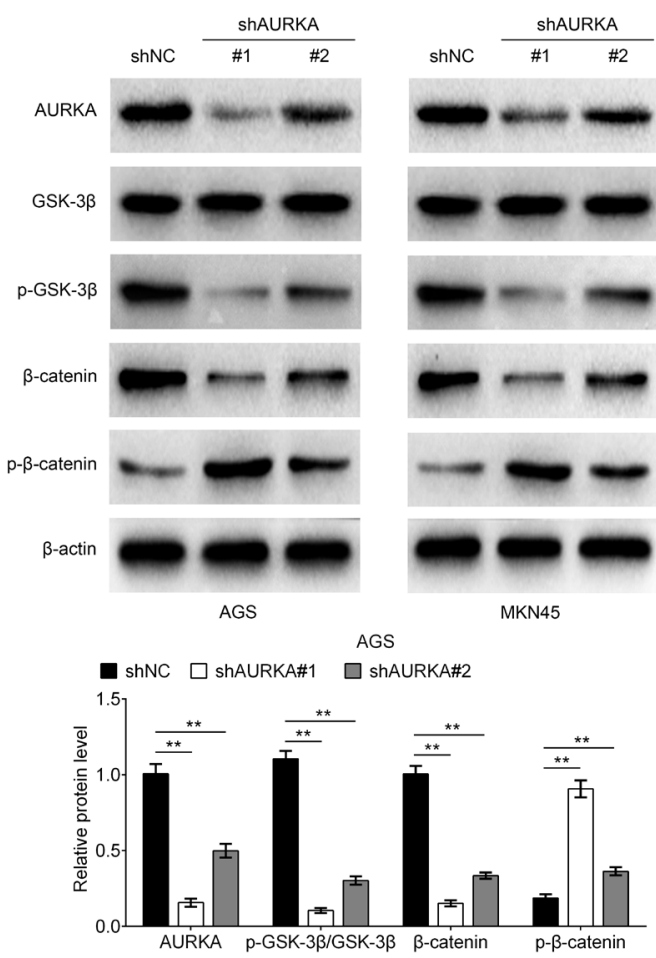

MKN45

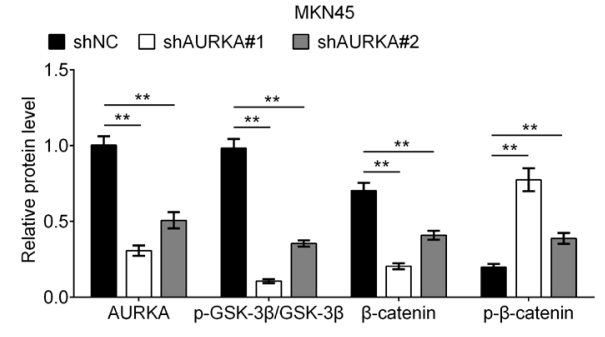

AURKA knockdown inhibited proliferation of gastric cancer cells

To investigate whether AURKA plays a role in cell proliferation, AURKA was knocked down in two gastric adenocarcinoma cell lines (AGS and MKN45) with two independent shRNA sequences (\#1 and\#2). The efficiency was confirmed at mRNA and protein levels. As shown in Fig. 4A (transcription) and Fig. 4B (protein expression), the level of AURKA decreased over $50 \%$ $(p<0.01$ vs. control) for both of the sequences, with \#1 inducing stronger reduction. GSK-3 $\beta$ is a major protein

C
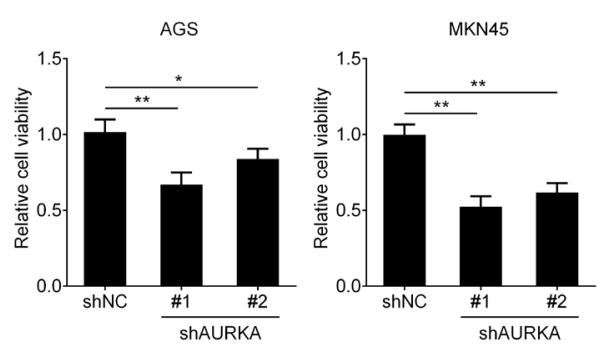

$\mathrm{D}$

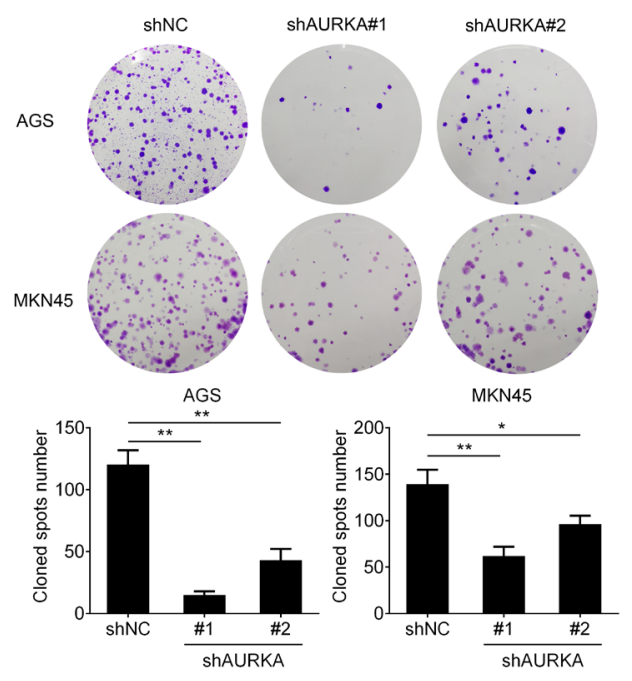

E
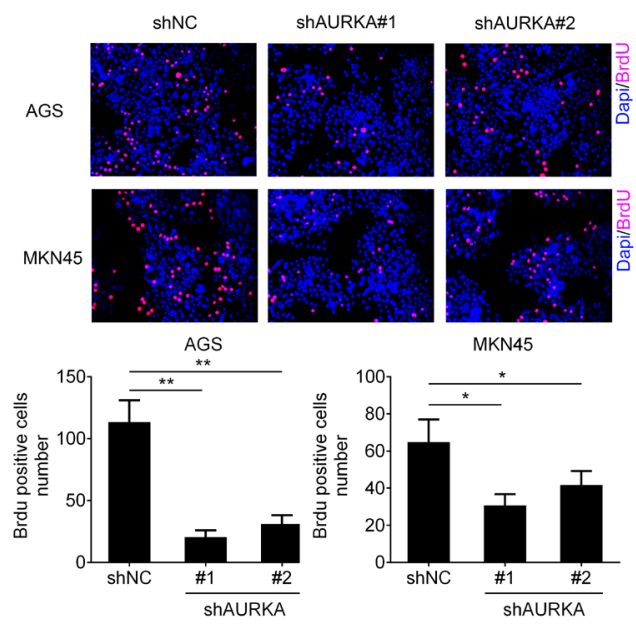

Figure 4. AURKA knockdown inhibited proliferation of gastric adenocarcinoma cells

(A) The efficiency of AURKA gene silencing in AGS and MKN45 cells with two shRNA sequences (\#1 and \#2) measured with qRT-PCR. (B) The level of AURKA, GSK-3 $\beta$ and $\beta$-catenin in AURKA knockdown cells analyzed with Western blot. (C) Cell viability of AGS and MKN45 cells with AURKA knockdown analyzed with MTT. (D) The growth of cancer cells (AGS and MKN45) with AURKA knockdown evaluated in colony formation assay. (E) Proliferation of cancer cells (AGS and MKN45) measured with BrdU labelling. $n=3,{ }^{* *} p<0.01$. 
kinase that regulates $\beta$-catenin phosphorylation. As previous reports confirmed that GSK-3 $\beta$ was a vital factor in gastric cancer, and AURKA promoted GSK-3 $\beta$ phosphorylation through direct binding and phosphorylating GSK-3 $\beta$ at Ser 9 (Dar et al., 2009), we analyzed the phosphorylation of GSK-3 $\beta$ and $\beta$-catenin with Western blot in AURKA knockdown cells. As shown in Fig. 4B, the ratio of phospho-GSK-3 $\beta$ to total GSK-3 $\beta$ (p-GSK$3 \beta /$ GSK-3 $\beta$ ) decreased after AURKA knockdown, and the ratio of phospho- $\beta$-catenin $/ \beta$-catenin increased,

A
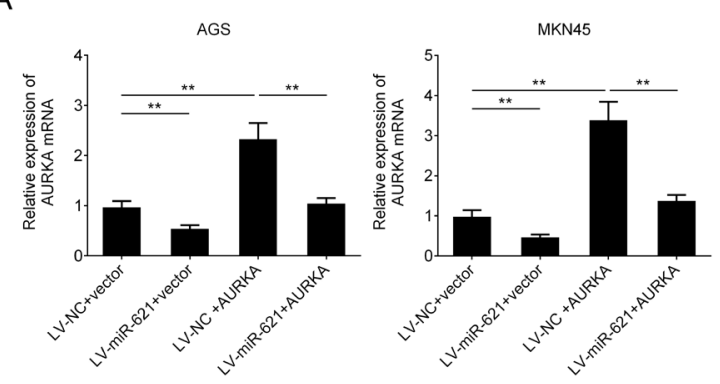

B
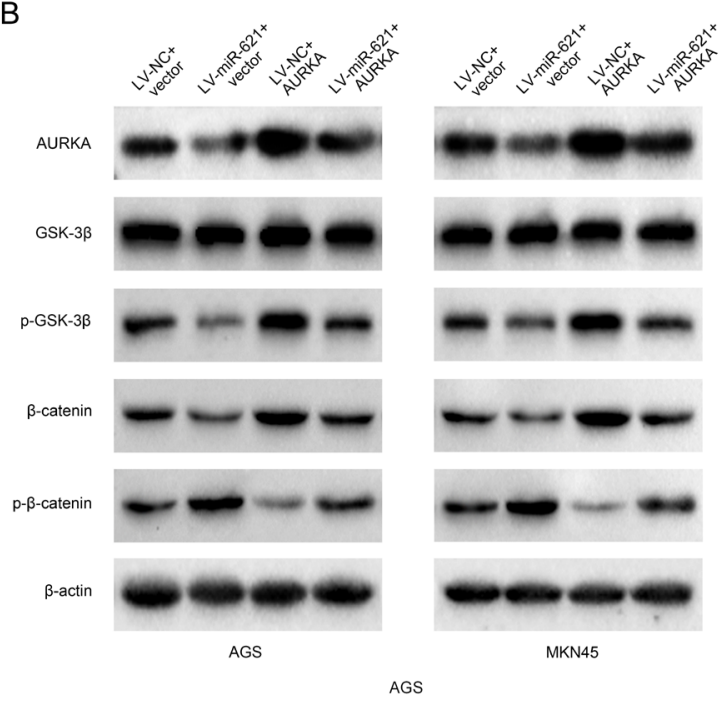

AGS

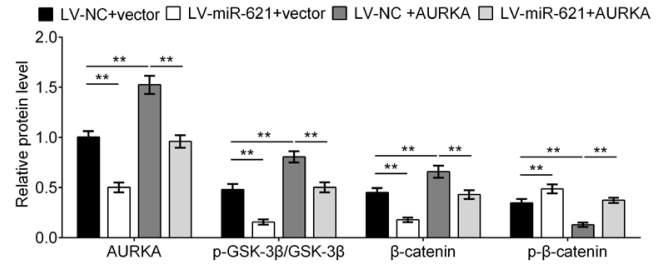

MKN45

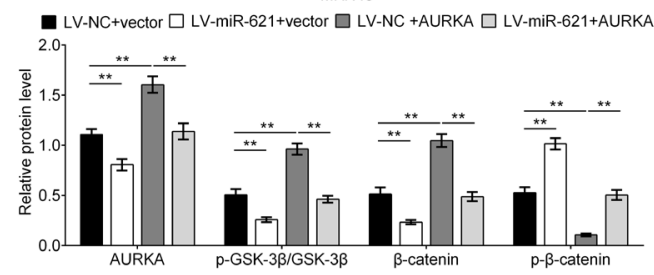

which confirmed that AURKA positively regulated the phosphorylation of GSK-3 $\beta$. Next, cell viability, colony formation, and proliferation were analyzed in $A U R K A$ knockdown cells (AGS and MKN45). Cell viability (Fig. 4C), colony formation (Fig. 4D), and proliferation (Fig. 4E) were all decreased after AURKA knockdown in both cell lines, and \#1 sequence induced stronger effects in AGS cells. These results indicated that AURKA knockdown inhibited proliferation of gastric adenocarci-

C
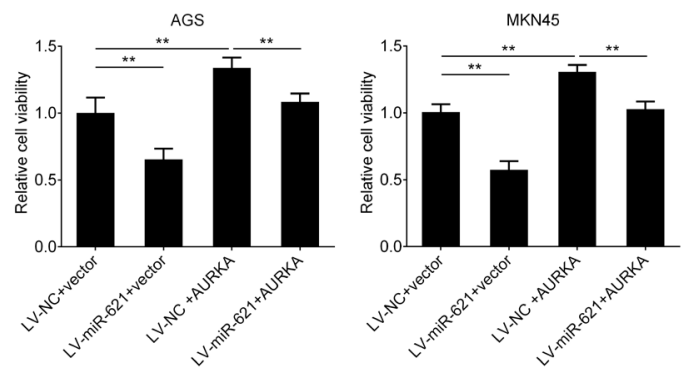

D

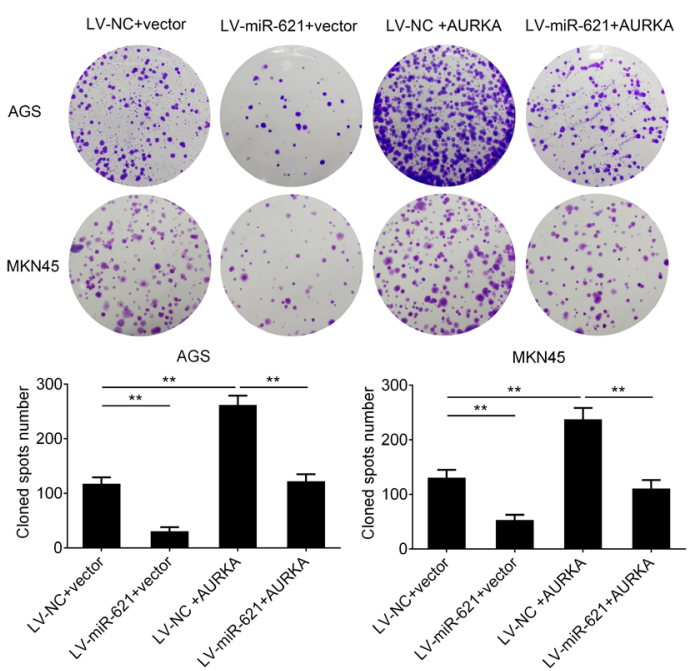

E
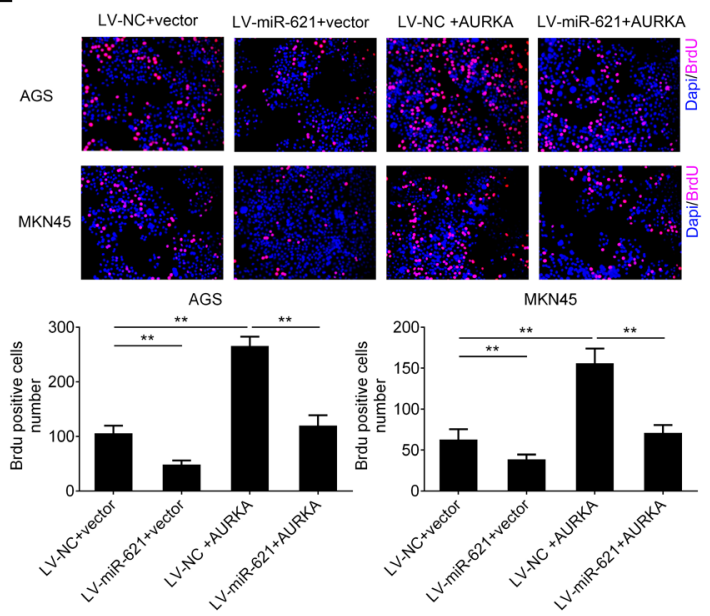

Figure 5. AURKA overexpression reversed the effect of miR-621 overexpression on the proliferation of gastric adenocarcinoma cells. (A) The transcription of AURKA in miR-621 and/or AURKA overexpressed cells (AGS and MKN45) measured with qRT-PCR. (B) The expression of AURKA in miR-621 and/or AURKA overexpressed cells (AGS and MKN45) measured with Western blot. (C) Cell viability of AGS and MKN45 cells with AURKA and/ or miR-621 overexpression analyzed with MTT. (D) The growth of cancer cells (AGS and MKN45) with AURKA and/ or miR-621 overexpression measured in colony formation assay. (E) Proliferation of cancer cells (AGS and MKN45) evaluated with BrdU labelling. $n=3,{ }^{* *} p<0.01$. 
noma cells, which was related to the GSK-3 $\beta / \beta$-catenin signaling pathway.

\section{AURKA overexpression reversed the effect of miR-621 overexpression on the proliferation of gastric adenocarcinoma cells}

Provided that AURKA was indeed a target of miR-621, and miR-621 negatively regulated the transcription of $A U R K A$, we hypothesized that the effects of miR-621 overexpression could be reversed by AURKA overexpression. Thus, the miR-621 and AURKA overexpression plasmids were co-transfected to gastric adenocarcinoma cells (AGS and MKN45). Similarly, cells overexpressing miR-621 had lower AURKA expression, while the co-transfection weakened or reversed this tendency (Fig. 5A). GSK-3 $\beta$ and $\beta$-catenin expression was also analyzed. MiR-621 overexpressing cells had a lower level of phospho-GSK-3 $\beta /$ GSK-3 $\beta$ ratio, and less phosphorylation of $\beta$-catenin. AURKA overexpression caused the opposite effect (Fig. 5B). Co-expression of miR-621 and $A U R K A$ weakened the effects of miR-621 or AUR$K A$ overexpression alone. Cell viability (Fig. 5C), colony formation (Fig. 5D), and proliferation (Fig. 5E) were all decreased significantly in miR-621 overexpressed cells, and these effects were all reversed by AURKA overexpression. These results demonstrated that AURKA overexpression could reverse the effects of miR-621 on the proliferation of gastric adenocarcinoma cells.

\section{DISCUSSION}

MiRNAs, small non-coding RNAs with the length of $18-22 \mathrm{bp}$, regulate various target genes by altering mRNA translation or stability. MiRNAs have been reported to play vital roles in many diseases, including many types of cancer (Farazi et al., 2011). For example, in patients with gastric cardia adenocarcinoma (GCA), the profile of miRNAs was different from that of the normal cells (Wang et al., 2018). A novel cancer-related miRNA, miR-621, was reported to have abnormal expression in several cancers, such as breast cancer, bladder cancer, and testicular cancer (Xue et al., 2016; Tao et al., 2020). Recently, researchers reported that miR-621 plays a critical role in gastric cancer. MiR-621 is downregulated in gastric cancer tissues, which is associated with clinical features. And in vivo xenograft model revealed that miR-621 overexpression inhibited tumor growth (Tao et al., 2020). In other cancers, miR-621 exerts other effects on cancer progression. For example, miR-621 could inhibit metastasis in bladder cancer through Wnt/ $\beta$-catenin signaling (Tian et al., 2019). MiR-621 also acts as a tumor radio- or chemo-sensitizer to radio- or chemo-therapy for breast cancer or hepatocellular carcinoma (Xue et al., 2016; Shao et al., 2019). In the present study, we confirm the effects of miR-621 in gastric adenocarcinoma. We showed that miR-621 was downregulated in human gastric adenocarcinoma and the decreasing level of miR-621 co-occurred with the larger tumor size. In addition, miR-621 overexpression inhibited cell viability, colony formation, and proliferation of gastric adenocarcinoma cells. These results were in accordance with the research of Tao that showed miR-621 overexpression inhibited tumor growth in vivo and in vitro (Tao et al., 2020).

Aurora kinases (AURKs) are members of serinethreonine kinases family essential for cell cycle. Aurora kinase A (AURKA) - the best-characterized member of the AURKs, is involved in the regulation of several oncogenic processes, including mitosis, centrosome maturation, chromosome segregation and alignment (Bolanos-Garcia, 2005). AURKA has been confirmed to be highly expressed in many types of tumors, including gastric cancer (Yan et al., 2016). Researchers preliminarily analyzed the expression profiles of histone modification genes in gastric cancer progression in patients, and found that $A U R K A$ and $H D A C 2$ genes were significantly overexpressed during gastric cancer development (Orenay-Boyacioglu et al., 2018). AURKA could induce epithelial-mesenchymal transition by regulating histone modification through Wnt/ $\beta$-catenin in gastric cancer. AURKA inhibitor decreased the phosphorylation of Akt1, and the activation of Akt and Wnt pathways. AURKA inhibitor also arrested the cells in the G2/M phase (Liu et al., 2016). Other reports also confirmed that inhibition of AURKA could reduce proliferation and survival of gastrointestinal cancer cells (WangBishop et al., 2019). AURKA promoted GSK-3 $\beta$ phosphorylation at Ser 9 through direct binding, leading to the decreased phosphorylation of $\beta$-catenin and thereby stabilizing the expression of $\beta$-catenin (Dar et al., 2009). Many previous studies indicated that AURKA was an important factor in gastric cancer process. Our research confirmed that $A U R K A$ knockdown inhibited viability, colony formation and proliferation of gastric adenocarcinoma cells. Bioinformatic analysis showed that AUR$K A$ was a target of miR-621, and AURKA positively regulated the phosphorylation of GSK-3 $\beta$. AURKA overexpression reversed the effects of miR-621 on the growth of gastric adenocarcinoma cells. The present results indicated that miRNA-621 exerted tumor suppressor function in gastric adenocarcinoma by targeting $A U R K A$. However, functional studies of miR-621 in vivo are required to further elucidate how miR-621 regulates gastric cancer, and this is one of the focuses of our future research.

In conclusion, current research showed that miR-621 was downregulated in gastric adenocarcinoma. MiR-621 inhibited cancer viability, colony formation, proliferation through targeting AURKA/GSK-3 $\beta$ pathway. AURKA overexpression reversed the effect of miR-621 on gastric adenocarcinoma cells. MiR-621 was an important tumor suppressor in gastric cancer and may be a promising target for cancer treatment.

\section{Acknowledgements}

Not applicable.

\section{Funding}

Not applicable.

\section{Competing interests}

The authors state that there are no conflicts of interest to disclose.

\section{Ethics approval}

Ethical approval was obtained from the Ethics Committee of the Affiliated Huaian No.1 People's Hospital of Nanjing Medical University.

\section{Statement of Informed Consent}

Written informed consent was obtained from a legally authorized representative(s) for anonymized patient information to be published in this article. 


\section{Availability of data and materials}

All data generated or analyzed during this study are included in this published article.

\section{Authors' contributions}

Xiao Han and Hongxue Liu designed the study, supervised the data collection, Xiaojun Tang analyzed the data, interpreted the data, Yao Zhao prepare the manuscript for publication and reviewed the draft of the manuscript. All authors have read and approved the manuscript.

\section{REFERENCES}

Bolanos-Garcia VM (2005) Aurora kinases. Int J Biochem Cell Biol 37: 1572-1577. https://doi.org/10.1016/j.biocel.2005.02.021

Dar AA, Belkhiri A, El-Rifai W (2009) The aurora kinase A regulates GSK-3beta in gastric cancer cells. Oncogene 28: 866-875. https://doi. org/10.1038/onc. 2008.434

Ding L, Xu Y, Zhang W, Deng Y, Si M, Du Y, Yao H, Liu X, Ke Y, Si J, Zhou T (2010) MiR-375 frequently downregulated in gastric cancer inhibits cell proliferation by targeting JAK2. Cell Res 20: 784-793. https://doi.org/10.1038/cr.2010.79

Farazi TA, Spitzer JI, Morozov P, Tuschl T (2011) miRNAs in human cancer. I Pathol 223: 102-115. https://doi.org/10.1002/path.2806

Izadpanahi P, Mohammadifard M, Tavakoli T, Abbasi N, Javadinia SA (2020) Effect of chemotherapy on fatty liver occurrence in breast and gastrointestinal cancer patients: a case-controlled study. Hepatitis Monthly 20: e97986. https://doi.org/10.5812/hepatmon.97986

Kontomanolis EN, Koukouli A, Liberis G, Stanulov H, Achouhan A, Pagkalos A (2016) MiRNAs: regulators of human disease. Eur J Gynaecol Oncol 37: 759-765. PMID: 29943916

Liu X, Li Z, Song Y, Wang R, Han L, Wang Q, Jiang K, Kang C, Zhang Q (2016) AURKA induces EMT by regulating histone modification through Wnt/beta-catenin and PI3K/Akt signaling pathway in gastric cancer. Oncotarget 7: 33152-33164. https://doi. org/10.18632/oncotarget.8888

Lu Q, Liu T, Feng H, Yang R, Zhao X, Chen W, Jiang B, Qin H, Guo X, Liu M, Li L, Guo H (2019) Circular RNA circSLC8A1 acts as a sponge of miR-130b/miR-494 in suppressing bladder cancer progression via regulating PTEN. Mol Cancer 18: 111. https://doi. org/10.1186/s12943-019-1040-0

Molina-Castro S, Pereira-Marques J, Figueiredo C, Machado JC, Varon C (2017) Gastric cancer: Basic aspects. Helicobacter 22 (Suppl 1). https://doi.org/10.1111/hel.12412

Orenay-Boyacioglu S, Kasap E, Gerceker E, Yuceyar H, Demirci U, Bilgic F, Korkmaz M (2018) Expression profiles of histone modification genes in gastric cancer progression. Mol Biol Rep 45: 22752282. https://doi.org/10.1007/s11033-018-4389-z
Rupaimoole R, Slack FJ (2017) MicroRNA therapeutics: towards a new era for the management of cancer and other diseases. Nat Rev Drug Discov 16: 203-222. https://doi.org/10.1038/nrd.2016.246

Shao Y, Song X, Jiang W, Chen Y, Ning Z, Gu W, Jiang J (2019) MicroRNA-621 acts as a tumor radiosensitizer by directly targeting SETDB1 in hepatocellular carcinoma. Mol Ther 27: 355-364. https://doi.org/https://doi.org/10.1016/j.ymthe.2018.11.005

Shin VY, Chu KM (2014) MiRNA as potential biomarkers and therapeutic targets for gastric cancer. World J Gastroenterol 20: 10432 10439. https://doi.org/10.3748/wjg.v20.i30.10432

Tan Z (2019) Recent advances in the surgical treatment of advanced gastric cancer: a review. Med Sci Monit 25: 3537-3541. https://doi. org/10.12659/msm.916475

Tao Y, Zhao Y, Peng Y, Ma X, Sun C, Xu K (2020) MicroRNA-621 inhibits the growth of gastric cancer cells by targeting SYF2. Arch Biochem Biophys 688: 108406. https://doi.org/https://doi. org/10.1016/j.abb.2020.108406

Tian H, Wang X, Lu J, Tian W, Chen P (2019) MicroRNA-621 inhibits cell proliferation and metastasis in bladder cancer by suppressing Wnt/ $\beta$-catenin signaling. Chem-Biol Int 308: 244-251. https://doi. org/https://doi.org/10.1016/j.cbi.2019.05.042

Wang-Bishop L, Chen Z, Gomaa A, Lockhart AC, Salaria S, Wang J, Lewis KB, Ecsedy J, Washington K, Beauchamp RD, El-Rifai W (2019) Inhibition of AURKA reduces proliferation and survival of gastrointestinal cancer cells with activated KRAS by preventing activation of RPS6KB1. Gastroenterol 156: 662-675. e667. https://doi. org/10.1053/j.gastro.2018.10.030

Wang J, Zhang H, Zhou X, Wang T, Zhang J, Zhu W, Zhu H, Cheng W (2018) Five serum-based miRNAs were identified as potential diagnostic biomarkers in gastric cardia adenocarcinoma. Cancer Biomark. 23: 193-203. https://doi.org/10.3233/cbm-181258

Wu C, Lyu J, Yang EJ, Liu Y, Zhang B, Shim JS (2018) Targeting AURKA-CDC25C axis to induce synthetic lethality in ARID1Adeficient colorectal cancer cells. Nat Commun 9: 3212. https://doi. org/10.1038/s41467-018-05694-4

Xue J, Chi Y, Chen Y, Huang S, Ye X, Niu J, Wang W, Pfeffer LM, Shao ZM, Wu ZH, Wu J (2016) MiRNA-621 sensitizes breast cancer to chemotherapy by suppressing FBXO11 and enhancing p53 activity. Oncogene 35: 448-458. https://doi.org/10.1038/onc.2015.96

Yan M, Wang C, He B, Yang M, Tong M, Long Z, Liu B, Peng F, Xu L, Zhang Y, Liang D, Lei H, Subrata S, Kelley KW, Lam EW, Jin B, Liu Q (2016) Aurora-A kinase: a potent oncogene and target for cancer therapy. Med Res Rev 36: 1036-1079. https://doi. org/10.1002/med.21399

Yepes S, López R, Andrade RE, Rodriguez-Urrego PA, LópezKleine L, Torres MM (2016) Co-expressed miRNAs in gastric adenocarcinoma. Genomics 108: 93-101. https://doi.org/10.1016/j. ygeno.2016.07.002

Zhang L, Hou Y, Ashktorab H, Gao L, Xu Y, Wu K, Zhai J, Zhang L (2010) The impact of C-MYC gene expression on gastric cancer cell. Mol Cell Biochem 344: 125-135. https://doi.org/10.1007/ s11010-010-0536-0

Zhou HY, Wu CQ, Bi EX (2019) MiR-96-5p inhibition induces cell apoptosis in gastric adenocarcinoma. World J Gastroenterol 25: 68236834. https://doi.org/10.3748/wjg.v25.i47.6823 